\title{
Fermi-Boltzmann statistics of neutrinos and relativistic effective degrees of freedom in the early universe
}

\author{
Jun IIZUKA* and Teruyuki KITABAYASHI \\ Department of Physics, Tokai University \\ 4-1-1 Kitakaname, Hiratsuka, Kanagawa, 259-1292, Japan \\ E-mail: 4btad003dmail.tokai-u.jp teruyukidtokai-u.jp
}

\begin{abstract}
We investigate the effects of the presence of non-pure fermionic neutrinos on the relativistic effective degrees of freedom in the early universe. The statistics of neutrinos is transformed continuously from Fermi-Dirac to Maxwell-Boltzmann statistics. We show that the relativistic degrees of freedom decreases with the deviation from pure Fermi-Dirac statistics of neutrinos if there are constant and large lepton asymmetries.
\end{abstract}

Flavor Physics \& CP Violation 2015

May 25-29, 2015

Nagoya, Japan

\footnotetext{
* Speaker.
} 


\section{Introduction}

Neutrinos have spin $1 / 2$ and it is natural that we understand the neutrinos obey purely FermiDirac statistics on the analogy. However, the possibility of the non-pure fermionic neutrinos is not excluded [ [ ㅁ]. Although the possibility of the pure bosonic neutrinos is excluded by double-beta decay [B] , it does not mean that the neutrinos are pure fermions. Moreover, properties of neutrinos are so different from other spin one half particles, e.g., tiny masses, large mixings, electrically neutral. Thus, the Pauli exclusion principle may be violated for neutrinos and they may possess mixed statistics.

In this paper, we investigate the effect of the presence of non-pure fermionic neutrinos on the relativistic effective degrees of freedom at temperature $T=0.5-500 \mathrm{GeV}$ (this paper is based on Ref.[䧃]). The assumption of violation of the Pauli principle makes problems but we put aside discussion of these problems and we restrict ourselves to the phenomenological approach. The large lepton asymmetries at MeV scale are almost excluded by the standard BBN cosmology [四]. However, the large lepton asymmetries at $\mathrm{GeV}$ scale are compatible with current observations [G].

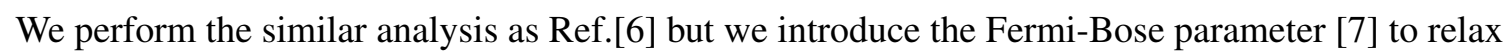
the statistics of neutrinos. We show that the relativistic degrees of freedom decreases with deviation from pure Fermi-Dirac statistics of neutrinos if there are constant and large lepton asymmetries at $\mathrm{GeV}$ scale universe.

\section{Statistics of neutrinos and relativistic effective degrees of freedom}

The distribution function of particle species $i$ is given by

$$
f_{i}=\frac{g_{i}}{e^{\left(E-\mu_{i}\right) / T}+\kappa_{i}}
$$

where $g_{i}, E, \mu_{i}$ and $T$ denote number of internal degrees of freedom, energy, chemical potential and temperature of particle $i$, respectively. The parameter $\kappa_{i}$ (we call it the Fermi-Bose parameter []] describes the continuous transition either from the Fermi-Dirac $\left(\kappa_{i}=1\right)$ to the Bose-Einstein $\left(\kappa_{i}=-1\right)$ via the Maxwell-Boltzmann $\left(\kappa_{i}=0\right)$ distributions. We assume that the spin one half particles $i$ obey "Fermi-Boltzmann" distribution with $0 \leq \kappa_{i} \leq 1$.

The number density $n_{i}$, energy density $\rho_{i}$, pressure $P_{i}$ and entropy density $s_{i}$ of particle species $i$ are obtained as follows [8]]:

$$
\begin{aligned}
n_{i} & =\frac{1}{2 \pi^{2}} \int_{m_{i}}^{\infty} E\left(E^{2}-m_{i}^{2}\right)^{1 / 2} f_{i} d E, \quad \rho_{i}=\frac{1}{2 \pi^{2}} \int_{m_{i}}^{\infty} E^{2}\left(E^{2}-m_{i}^{2}\right)^{1 / 2} f_{i} d E, \\
P_{i} & =\frac{1}{6 \pi^{2}} \int_{m_{i}}^{\infty}\left(E^{2}-m_{i}^{2}\right)^{3 / 2} f_{i} d E, \quad s_{i}=\frac{\rho_{i}+P_{i}-\mu_{i} n_{i}}{T},
\end{aligned}
$$

According to Stuke, et.al.[G], we define the general relativistic effective degrees of freedom $g_{*}=$ $g_{*}\left(m_{i}, \kappa_{i}, \mu_{i}\right)$ for any $m_{i}, \kappa_{i}$ and $\mu_{i}$ as follows

$$
g_{*}=\frac{30}{\pi^{2} T^{4}} \rho,
$$

where $\rho=\sum_{i} \rho_{i}\left(m_{i}, \kappa_{i}, \mu_{i}\right)$. 
To estimate the total energy density $\rho$, the chemical potentials of all particle species $\mu_{i}$ should be determined [可]. The beta-decay of down quark $d$ via weak interactions provides $\mu_{u}+\mu_{f}=$ $\mu_{d}+\mu_{v_{f}}$ where $f=e, \mu, \tau$. We assume that $\mu_{u}=\mu_{c}=\mu_{t}$ and $\mu_{d}=\mu_{s}=\mu_{b}$ for quarks. All gauge bosons and Higgs boson have vanishing chemical potentials. The five independent chemical potentials $\mu_{v_{e}}, \mu_{v_{\mu}}, \mu_{v_{\tau}}, \mu_{u}, \mu_{d}$ are uniquely determined by the following five conservation laws:

$$
s q=-\sum_{i=e, \mu, \tau} n_{i}+\frac{2}{3} \sum_{i=u, c, t} n_{i}-\frac{1}{3} \sum_{i=d, s, b} n_{i}, \quad s b=\frac{1}{3} \sum_{i=q u a r k s} n_{i}, \quad s \ell_{f}=n_{f}+n_{v_{f}}
$$

where $s=\sum_{i} s_{i}$ and $q$ denote total entropy density and electric charge of the universe, respectively. Here, baryon number $b$ and lepton flavour number $\ell_{f}$ are defined as

$$
b=\frac{n_{b}-n_{\bar{b}}}{s}, \quad \ell_{f}=\frac{n_{f}-n_{\bar{f}}+n_{v_{f}}-n_{\bar{v}_{f}}}{s},
$$

where $n_{b}$ and $n_{\bar{b}}$ denote the number densities of baryons and anti-baryons, respectively. From charge neutrality of the universe, we assume $q=0$. The baryon number is fixed to $b=9 \times 10^{-11}$ [四]. The neutrino oscillations may ensure the equilibration of the lepton flavour numbers in the early universe [ए]], we assume that all lepton flavour numbers are the same $\ell=\ell_{e}=\ell_{\mu}=\ell_{\tau}$. Since the measurements of the lepton flavour numbers in the early universe are not established, we keep $\ell$ as free parameters. We assume that all neutrino masses are same $m_{v}=1 \mathrm{eV}$ [प]].

The Fermi-Bose parameters $\kappa_{i}$ of standard model particles $i$ are assigned as follows: $0 \leq \kappa_{v} \leq 1$ for neutrinos $v_{f}$ (we assume that $\kappa_{v}=\kappa_{v_{e}}=\kappa_{v_{\mu}}=\kappa_{v_{\tau}}$ ), $\kappa_{\ell}=\kappa_{q}=+1$ for charged leptons $\ell$ and quarks $q, \kappa_{\gamma, W, Z, g}=\kappa_{H}=-1$ for gauge bosons $\gamma, W, Z, g$ and Higgs boson $H$. In this case

$$
g_{*}=g_{*}^{m_{i}=\mu_{i}=0}+\Delta g_{*}\left(\kappa_{v}=1, \mu_{i} \neq 0\right)+\Delta g_{*}\left(\kappa_{v} \neq 1, \mu_{i} \neq 0\right),
$$

where $g_{*}^{m_{i}=\mu_{i}=0}=\sum_{i=\text { bosons }} g_{i}+(7 / 8) \sum_{i=\text { fermions }} g_{i}$ denotes well known relativistic effective degrees of freedom [ [8] and $\Delta g_{*}\left(\kappa_{v}=1, \mu_{i} \neq 0\right)$ denotes the deviation from $g_{*}^{m_{i}=\mu_{i}=0}$ due to the non-zero lepton asymmetries which has been reported [目]. The 3rd term in R.H.S in Eq.([2.7), $\Delta g_{*}\left(\kappa_{v} \neq 1, \mu_{i} \neq 0\right)$ is the new contribution on the relativistic effective degrees of freedom $g_{*}$.

\section{Numerical estimation}

Figure $\square$ shows the dependence of the relativistic effective degrees of freedom $g_{*}$ on temperature $T$. The left figure shows the numerical solution for $\ell=0, \ell=0.01$ and $\ell=0.03$ cases. There are three curves correspond to $\kappa_{v}=1,0.5,0$ for $\ell=0.03$. For $\ell=0.01$ and $\ell=0$, these three curves almost overlap with each other. The right figure shows the $\ell=0$ case in more detail. The energy density of pure bosons $\left(\kappa_{i}=-1\right)$ is larger than the energy density of pure fermions $\left(\kappa_{i}=1\right)$ by the factor $8 / 7[[]]$. Thus one may expect, if neutrinos have non-pure Fermi-Dirac statistics $\left(\kappa_{v} \neq 1\right)$, their lager energy density would correspond to an increase of the effective degrees of freedom $g_{*}$ in Eq.(2.4). Indeed, if there is no lepton asymmetries (right figure), relativistic effective degrees of freedom for pure Maxwell-Boltzmann neutrinos case is lager than it for pure Fermi-Dirac neutrinos case; $g_{*}^{\ell=0, \kappa_{v}=0}>g_{*}^{\ell=0, \kappa_{v}=1}$. However, if there is large lepton asymmetries, such as $\ell=0.03$ (left figure), the relativistic effective degrees of freedom for pure Maxwell-Boltzmann neutrinos case is smaller than it for pure Fermi-Dirac neutrinos case; $g_{*}^{\ell=0.03, \kappa_{\nu}=0}<g_{*}^{\ell=0.03, \kappa_{v}=1}$. 

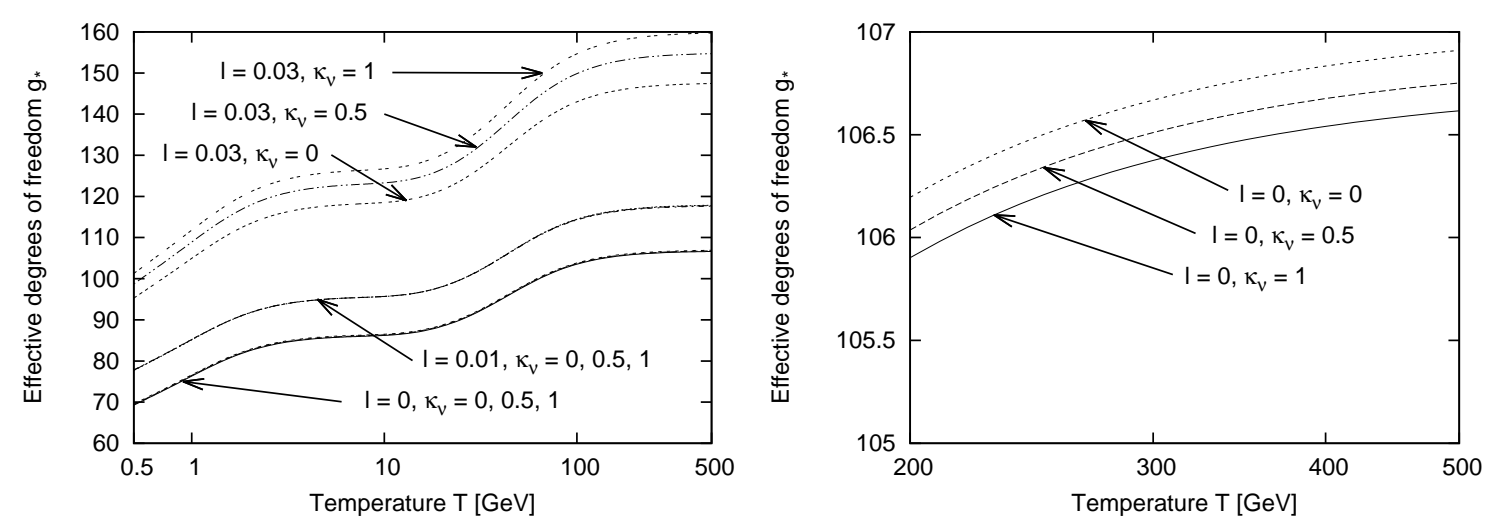

Figure 1: Dependence of the relativistic effective degrees of freedom $g_{*}$ on temperature $T$. The left figure shows the numerical solution for $\ell=0, \ell=0.01$ and $\ell=0.03$ cases. The right figure shows the $\ell=0$ case in more detail.
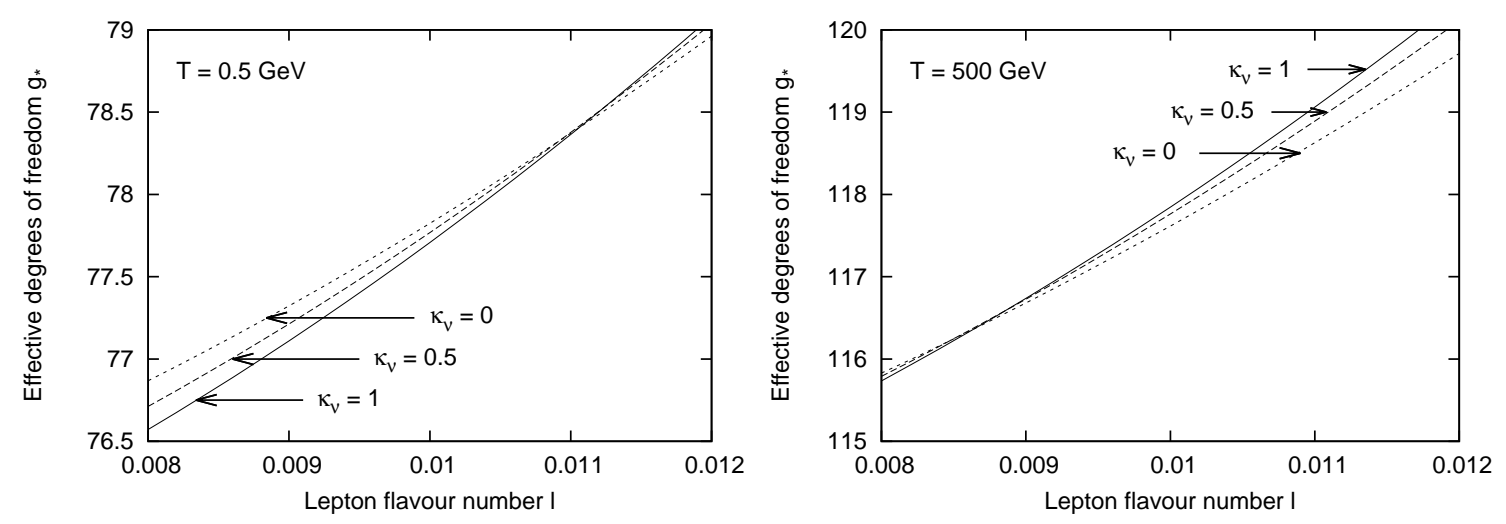

Figure 2: The dependence of the relativistic effective degrees of freedom $g_{*}$ on lepton flavour number $\ell$ for $T=0.5 \mathrm{GeV}$ (left) and for $T=500 \mathrm{GeV}$ (right).

Figure $\square$ shows the dependence of the relativistic effective degrees of freedom $g_{*}$ on lepton flavour number $\ell$. The left figure shows the result for $T=0.5 \mathrm{GeV}$ while the right figure shows the same but for $T=500 \mathrm{GeV}$. In the case of $T=0.5 \mathrm{GeV}, g_{*}^{\kappa_{v}=1}$ and $g_{*}^{\kappa_{v}=0}$ are equivalent at $\ell^{e q} \sim 0.0112$. In the case of $T=500 \mathrm{GeV}$, the equivalent lepton flavour number is $\ell^{e q} \sim 0.0087$. In the range of $0.5 \leq \mathrm{T}[\mathrm{GeV}] \leq 500$, the equivalent lepton flavour number to be $0.0087 \lesssim \ell^{e q} \lesssim 0.0112$ and $\ell^{e q}$ decreases with the increasing temperer $T$.

Recall that the relativistic effective degrees of freedom $g_{*}$ increases with the energy density $\rho$ and this energy density increases with distribution function $f_{i}=g_{i} /\left[e^{\left(E-\mu_{i}\right) / T}+\kappa_{i}\right]$. If there is no lepton asymmetry $(\ell=0)$ then the chemical potential of neutrinos vanish $\mu_{v}=0$ and the small $\kappa_{v}$ induces large $f_{v}$ as well as large $g_{*}$. Moreover, if there are non-zero lepton asymmetry $\ell \neq 0$, the small $\kappa_{v}$ still induces large $g_{*}$ as long as the chemical potential $\mu_{v}$ is constant. In other word, if the chemical potential $\mu_{v}$ is independent of $\kappa_{v}$ and is constant, the relation of $g_{*}^{\mu_{v}=\text { const, } \kappa_{v}=0}>$ $g_{*}^{\mu_{v}=c o n s t, \kappa_{v}=1}$ is obtained. However, if the Fermi-Bose parameter $\kappa_{v}$ change continuously from 
$\kappa_{v}=1$ to $\kappa_{v} \neq 1$, even if the lepton flavour number is constant, the chemical potential $\mu_{v}$ is no longer constant with $\kappa_{v}$. The small $\kappa_{v}$ induces large $f_{v}$ while the small $\mu_{v}$ induces small $f_{v}$. In this case the relation of $g_{*}^{\mu_{v}=\text { const }, \kappa_{v}=0}>g_{*}^{\mu_{v}=\text { const }, \kappa_{v}=1}$ is no longer guaranteed and $g_{*}^{\mu_{v} \neq c o n s t, \kappa_{v}=0}<$ $g_{*}^{\mu_{v} \neq c o n s t, \kappa_{v}=1}$ may be allowed. If the effect of $\mu_{v}$ on $f_{v}$ is dominant, the energy density will be reduced. This is the reason why we encounter $g_{*}^{\ell=0.03, \kappa_{v}=0}<g_{*}^{\ell=0.03, \kappa_{v}=1}$ in the Figure प.

\section{Conclusions}

We have investigated the effect of the presence of non-pure fermionic neutrinos on the relativistic effective degrees of freedom at temperature $0.5-500 \mathrm{GeV}$. It turned out that the relativistic degrees of freedom decreases with deviation from pure Fermi-Dirac statistics of neutrinos if there are constant and large lepton asymmetries at $\mathrm{GeV}$ scale universe.

The relativistic effective degrees of freedom increases with the energy density and this energy density increases with distribution function of particle species $i ; f_{i}=g_{i} /\left[e^{\left(E-\mu_{i}\right) / T}+\kappa_{i}\right]$. If the Fermi-Bose parameter $\kappa_{v}$ change continuously from $\kappa_{v}=1$ to $\kappa_{v} \neq 1$, even if the lepton flavour number is constant, the chemical potential $\mu_{v}$ is no longer constant with $\kappa_{v}$. The small $\kappa_{v}$ induces large $f_{v}$ while the small $\mu_{v}$ induces small $f_{v}$. If the effect of $\mu_{v}$ on $f_{v}$ is dominant, the energy density and relativistic effective degrees of freedom are reduced.

\section{References}

[1] A. D. Dolgov and A. Yu. Smirnov, Possible violation of the spin-statistics relation for neutrinos: Cosmological and astrophysical consequences, Phys. Lett. B 621, 1 (2005).

[2] W. Tornow, Search for a bosonic component in the neutrino wave function, Nucl. Phys. A 844, 57c (2010).

[3] A. S. Barabash, A. D. Dolgov, R. Dvornickỳ, F. Šimkovic and A. Yu. Smirnov, Statistics of neutrinos and the double beta decay, Phys. Lett. B 783, 90 (2007).

[4] J. Iizuka and T. Kitabayashi, Fermi-Boltzmann statistics of neutrinos and relativistic effective degrees of freedom in the early universe, Mod. Phys. Lett. A 30, 1550003 (2015).

[5] G. Mangano, G. Miele, S. Pastor, O. Pisanti and S. Sarikas, Updated BBN bounds on the cosmological lepton asymmetry for non-zero $\theta_{13}$, Phys. Lett. B 708, 1 (2012).

[6] M. Stuke, D. J. Schwarz and G. Starkman, WIMP abundance and lepton (flavour) asymmetry, JCAP 03, 040 (2012).

[7] A. D. Dolgov, S. H. Hansen and A. Yu. Smirnov, Neutrino statistics and big bang nucleosynthesis, JCAP 06, 004 (2005).

[8] E. W. Kolb and M. S. Turner, The Early Universe, Addison-Wesley, (1990).

[9] P. A. R. Ade, et. al. (Planck Collaboration), Planck 2013 results. XVI. Cosmological parameters, Astron. Astrophys. 571, A16 (2014).

[10] A. D. Dolgov, S. H. Hansen, S. Pastor, S. T. Petcov, G. G. Raffelt and D. V. Semikoz, Cosmological bounds on neutrino degeneracy improved by flavor oscillations, Nucl. Phys. B 632, 363 (2002).

[11] E. Giusarma, E. D. Valentino, M. Lattanzi, A. Melchiorri and O. Mena, Relic neutrinos, thermal axions, and cosmology in early 2014, Phys. Rev. D 90, 043507 (2014). 